\title{
FIRST BEAM FROM THE TRASCO INTENSE PROTON SOURCE (TRIPS) AT INFN-LNS
}

\author{
G. Ciavola, L.Celona, S. Gammino, F. Chines, S. Passarello, INFN-LNS, Catania, Italy \\ C. Campisano, HITEC, Catania, Italy
}

\begin{abstract}
The TRASCO intense proton source (TRIPS) has been installed at INFN-LNS in May 2000 and is now operational. The paper describes the design of the source along with the first results obtained and the next developments. The source has been able to deliver more than $20 \mathrm{~mA}$ of protons from a $5 \mathrm{~mm}$ hole at $65 \mathrm{kV}$ extraction voltage, largely above the minimum requested current density. The optimisation of the source is under way, with special care to the reliability and satisfactory results have been achieved.
\end{abstract}

\section{INTRODUCTION}

The TRASCO Project is a R\&D program which goal is the design of an Accelerator Driving System for nuclear waste transmutation. The high current $\mathrm{cw}$ proton linear accelerator will drive a subcritical system to transmutate nuclear wastes.[1]

The accelerator design is shared between different INFN laboratories [2] and the LNS is in charge of the source design and construction.

The proton source TRIPS is a high intensity microwave source, which goal is the production of a minimum proton current of $35 \mathrm{~mA}$ at the RFQ entrance, with a rms normalized emittance lower than $0.2 \pi \cdot \mathrm{mm} \cdot \mathrm{mrad}$ for an operating voltage of $80 \mathrm{kV}$.

With respect to the other sources for high intensity applications, some new features have been added:

- the microwave matching system has been improved by means of an appropriate matching transformer;

- a system to move the coils on-line has been realized;

- the extraction system has been optimised with the aim to increase the source availability and reliability, in order to meet the requirement of a driver for an ADS system.

\section{THE SOURCE DESIGN}

The design of TRIPS is shown in figure 1. The microwave power obtained with a $2.45 \mathrm{GHz}-2 \mathrm{~kW}$ magnetron is coupled to the cylindrical water cooled OFHC copper plasma chamber $(100 \mathrm{~mm}$ long and $90 \mathrm{~mm}$ in diameter) through a circulator, a four stub automatic tuning unit and a maximally flat matching transformer. The microwave pressure window is placed behind a water-cooled bend in order to avoid any damage due to the back-streaming electrons (fig. 1).

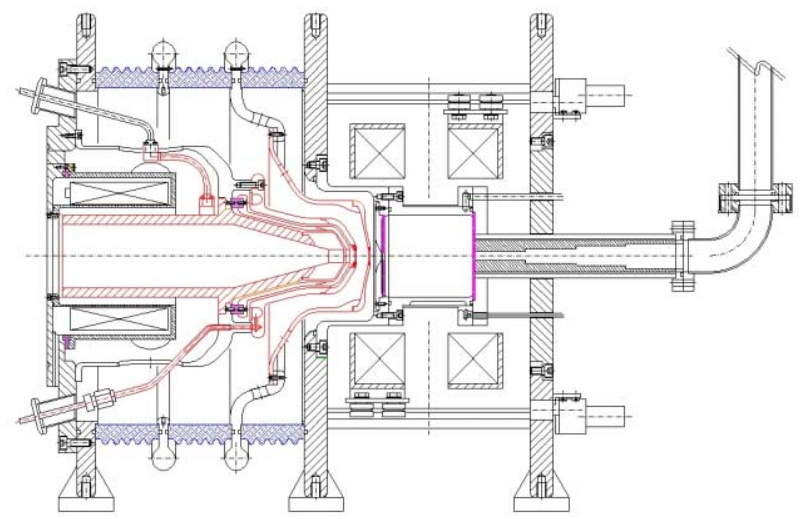

Figure 1: The TRIPS ion source

Two coils, independently on-line movable and energized with separate supplies, allow to vary the position of the electron cyclotron resonance (ECR) zones in the chamber and to produce the desired magnetic field configuration. The design have been aimed to simplify the maintenance especially in the extraction zone.

The maximally flat matching transformer [3] optimizes the coupling between the microwave generator and the plasma chamber (fig. 2). The transformer realizes a progressive match between the two impedances $\mathrm{Z}_{0}$ and $\mathrm{Z}_{5}$ and concentrates the electric field at the center of the plasma chamber (in our design the field enhancement ratio is around 2). The overall result is a significant increase of the extracted current density as we have observed during the commissioning of the source MIDAS2 which uses the same type of matching unit.[4]

The extraction geometry of the TRIPS source is the result of a study on the SILHI extraction system carried out in collaboration with the CEA team and with LANL team [5]. Different topologies have been investigated in order to increase the reliability. The main configurations studied were the tetrode (used at LANL) and the pentode (currently used at CEA on SILHI); all the simulations have been carried out with the AXCEL code, being the results cross checked with the IGUN code and with the PBGUN code.

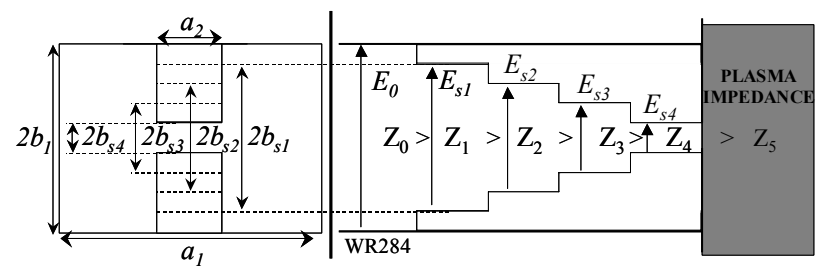

Figure 2: A sketch of the matching transformer 


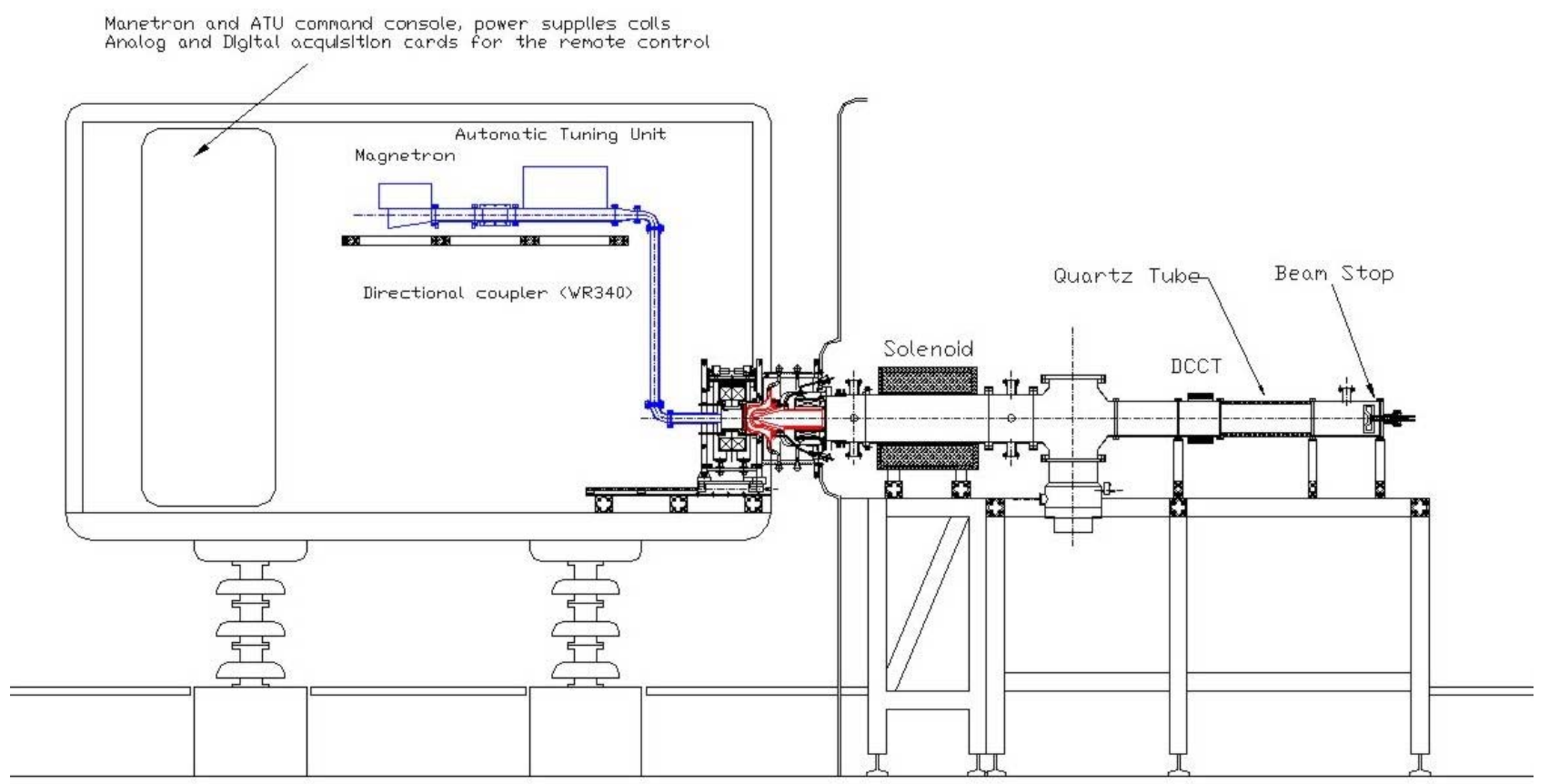

Figure 3: The experimental set-up.

The final design of the extraction system of TRIPS benefits of this experience: being the operational voltage of TRIPS just $80 \mathrm{kV}$ (instead of $95 \mathrm{kV}$ of SILHI), the extraction system was redesigned and the electrode geometry has been varied.

The gaps, the voltage and the extraction holes have been modified in order to reduce the length of the extraction zone where the beam is uncompensated and to reduce the aperture-lens effect. Beam dynamics simulations have been performed with the AXCEL code and the results have been cross-checked with the IGUN code. Rms normalized emittance below $0.2 \pi \mathrm{mm} \mathrm{mrad}$ (including the beam halo) have been calculated [6].

\section{EXPERIMENTAL RESULTS}

Fig. 3 shows the experimental set-up: the $100 \mathrm{kV}$ high voltage platform (fig.4) and the first section of the low energy beam transfer line (LEBT) devoted to the beam analysis and characterization (fig.5) are now fully operational after some improvements to the water cooling circuit. The LEBT line consists of a first current transformer (DCCT1), a solenoid to provide the necessary beam focusing, of a four sector ring to measure beam misalignments and in homogeneities, of a second current transformer (DCCT2) and of a $10 \mathrm{~kW}$ beam stop (BS) to measure the beam current.

The first operations of TRIPS have been performed at an extraction voltage of $65 \mathrm{kV}$ (instead of $80 \mathrm{kV}$ requested) and with an extraction aperture of $5 \mathrm{~mm}$ (instead of $8 \mathrm{~mm}$ of the final design), in order to work at relatively low currents to verify the functionality of all the ancillary equipment and to find the optimum parameters of the source. In this configuration a proton beam of 20 $\mathrm{mA}$ can be routinely generated (the Child-Langmuir limit for the current configuration is around $28 \mathrm{~mA}$ ) and then transported through the LEBT line with high transmission. Typically more than $90 \%$ of the HV drain current is detected by the DCCT2 and the same current value is measured by the beam stop. We have calculated that the beamline transmission is almost $100 \%$ which means that the proton fraction is anyhow above $90 \%$ if the discharge power exceeds $300 \mathrm{~W}$. Such excellent result is obtained by means of an accurate rf matching: the microwave coupling with the matching transformer and the automatic tuning unit works fine, permitting to have very low values of reflected power (below 5\%) and a high electric field on the axis, thus increasing either the proton fraction and the current density, above $100 \mathrm{~mA} / \mathrm{cm}^{2}$. The best results have been obtained through the optimisation of the coils parameters (positions and currents); the moving solenoids have permitted to optimise the ionisation process by changing easily the magnetic field profile (fig. 6 shows the best one).

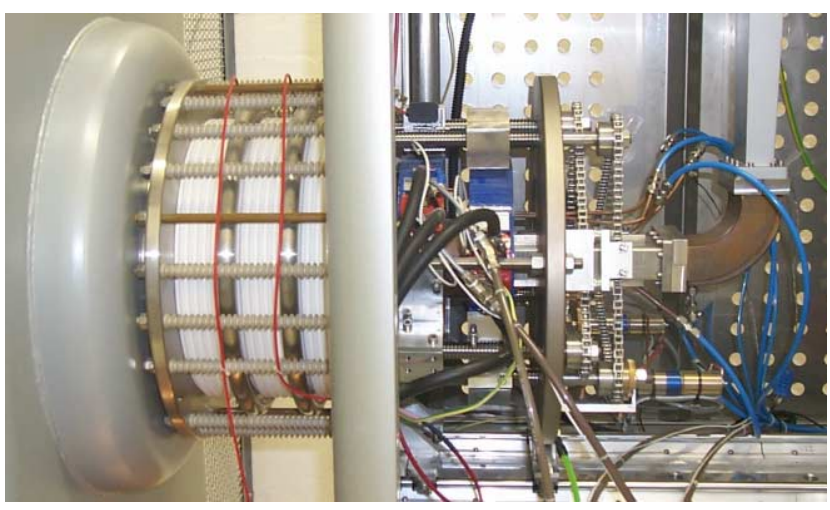

Figure 4: The TRIPS ion source on the $100 \mathrm{kV}$ platform 


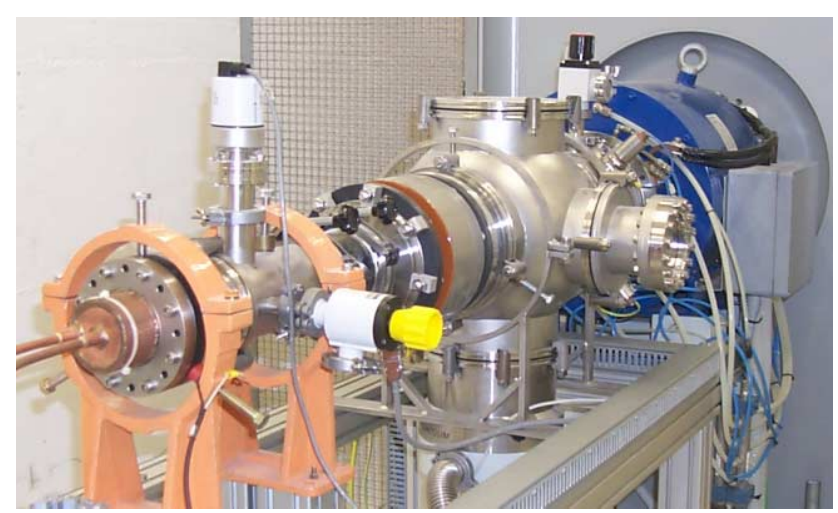

Figure5: The LEBT line.

At this moment a temporary solenoid supply is used, which can only focus proton, then the behavior of $\mathrm{H}_{2}^{+}$and $\mathrm{H}_{3}^{+}$will be subject of study in the next future.

Finally we have investigated the more stringent request concerning the reliability with a 24 hours test (see tab. 1).

Table 1: TRIPS parameters during the test

\begin{tabular}{|l|c|}
\hline \multicolumn{1}{|c|}{ Parameter } & $65 \mathrm{KV}$ \\
\hline Extraction voltage & $32.5 \mathrm{kV}$ \\
\hline Puller voltage & $-2 \mathrm{kV}$ \\
\hline Repeller voltage & $480 \mathrm{~W}$ \\
\hline Discharge power & $15 \mathrm{~mA}$ \\
\hline Beam current & $8.510^{-6} \mathrm{mbar}$ \\
\hline Pressure (extract.area) & \\
\hline
\end{tabular}

After the conditioning (a couple of hours), the source have been operating without discharge for 24 hours.

The source have not required any particular tuning during 24 hours, except for a few variations of the inlet hydrogen pressure in the plasma chamber. This is really an important parameter that has a strong influence on the stability of the beam current; for this reason a feedback system on the mass flow of the inlet gas is under study. Moreover, we have chosen a magnetic field profile that gives a quiet plasma with the two ECR zones inside the plasma chamber and then we have measured the beam current vs. discharge power (fig.7) at an extraction voltage of $65 \mathrm{kV}$. It must be remarked that the source can produce vey high current with quite low rf power.

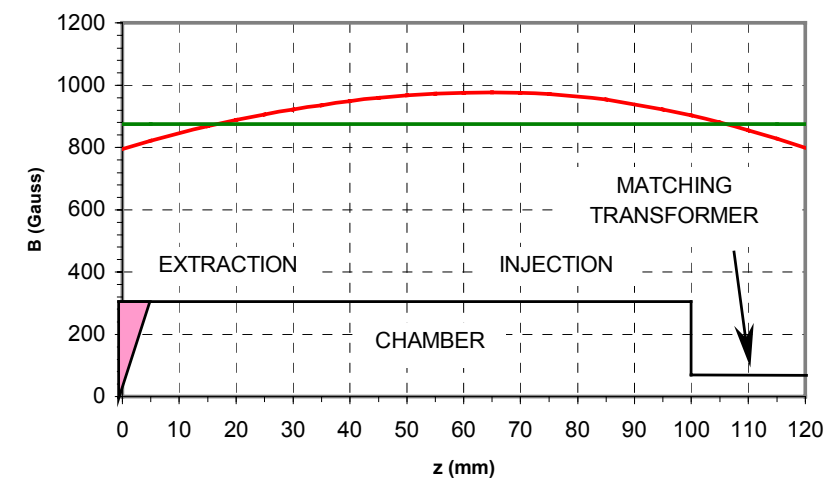

Figure 6: The magnetic profile used in the reliability test.

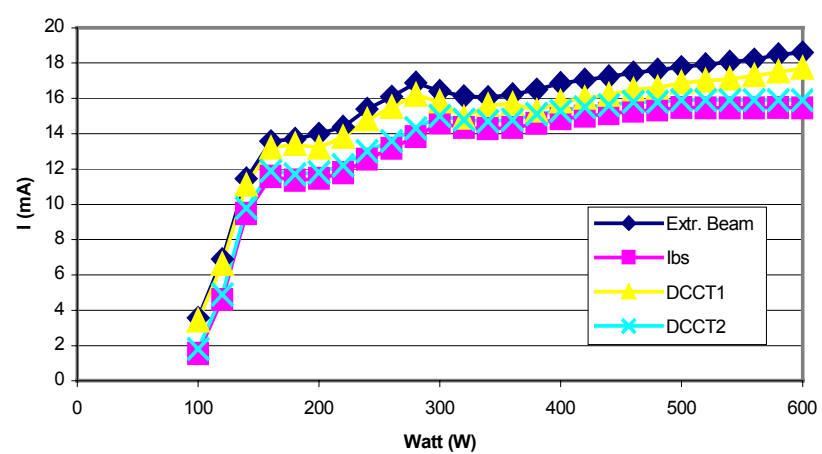

Figure 7: Beam currents vs. discharge power.

\section{CONCLUSION AND FUTURE DEVELOPMENTS}

In table 2 the status of the source is compared with the requirements. The beam energy will be upgraded in the next few weeks, after that the characterization will be completed. Then the $8 \mathrm{~mm}$ extraction hole will replace the $5 \mathrm{~mm}$ one which is now used and the final reliability test (over one or two weeks) and the emittance measurements will be done.

An interceptive beam emittance measurement station from the CEA/Saclay, used at the SILHI source, will be moved to LNS for the beam emittance study, in order to complete the commissioning in 2002.

Table 2: TRIPS status

\begin{tabular}{|l|c|c|}
\hline & Requirement & Status \\
\hline Beam energy & $80 \mathrm{keV}$ & $65 \mathrm{keV}$ \\
\hline Proton current & $35 \mathrm{~mA}$ & $20 \mathrm{~mA}$ \\
\hline Reliability $(24 \mathrm{~h})$ & $\approx 100 \%$ & $100 \% @ 15 \mathrm{~mA}$ \\
\hline Beam emittance & $\leq 0.2 \pi \mathrm{mmmrad}$ & --- \\
\hline
\end{tabular}

\section{REFERENCES}

[1] F. Carminati, R. Klapish, J. P. Revol, Ch. Roche, J. A. Rubio, and C. Rubbia, Report No. CERN/AT/93-47.

[2] The TRASCO_AC Group, Status of the high current proton accelerator for the TRASCO program. Report INFN/TC-00/23.

[3] L. Celona, G. Ciavola, S. Gammino, Rev. Sci. Instum 69 (2), 1113.

[4] G. Ciavola, L. Celona, S. Gammino, S. Marletta, C. Campisano, Proceedings of EPAC 2000, Vienna, Austria, 1601.

[5] Third activity report on SILHI ion source. Report CEA DAPNIA/SEA/IPHI 99-52.

[6] L. Celona, G. Ciavola, S. Gammino, R. Gobin, R. Ferdinand, Rev. Sci. Instrum. 71 (2), 771. 